\title{
MiR-802 alleviates lipopolysaccharide-induced acute lung injury by targeting Peli2
}

\author{
Qinghai You ${ }^{1} \cdot$ Jinmei Wang $^{2} \cdot$ Dan Jia $^{3} \cdot$ Lijuan Jiang $^{4} \cdot$ Yuanmin Chang ${ }^{5} \cdot$ Wenmei Li $^{5}$
}

Received: 10 July 2019 / Revised: 6 October 2019 / Accepted: 22 October 2019 / Published online: 6 November 2019

(c) Springer Nature Switzerland AG 2019

\begin{abstract}
Introduction Acute respiratory distress syndrome (ARDS) is a life-threatening medical condition. It is characterized by serious lung inflammation or injury. Characterizing novel miRNAs implicated in ARDS pathogenesis may provide new therapeutic strategy for managing ARDS.

Methods We employed LPS-induced lung injury model to profile miRNAs associated with ARDS. We isolated one miRNA candidate and characterized its role in lipopolysaccharide (LPS)-induced proinflammatory cytokine production in lung macrophages. We further evaluated its functional role in ARDS model by assessing histological change, neutrophil activation, tissue permeability and tumor necrosis factor alpha (TNF $\alpha$ ) production. We also characterized its downstream target using luciferase assay, Western blotting, enzyme-linked immunosorbent assay and cell inflammation assay.

Results Microarray profiling revealed miR-802 was significantly downregulated in ARDS mouse model. LPS-induced miR802 downregulation was confirmed in lung macrophages. Overexpression of miR-802 significantly suppressed LPS-induced inflammatory cytokine production in vitro and alleviates LPS-induced acute lung injury in vivo. Peli2 was identified as a downstream target of miR-802 and found upregulated in ARDS model. Overexpressing Peli2 abolished the antagonizing effect of miR-802 on LPS-mediated inflammatory response.

Conclusion MiR-802 carried a protective role against LPS-induced acute lung injury by downregulating Peli2. MiR-802/ Peli2 axis may act as intervening targets to manage ARDS.
\end{abstract}

Keywords Acute respiratory distress syndrome $\cdot \operatorname{miR} 802 \cdot$ Sepsis $\cdot$ Peli2 $\cdot$ Lung injury

Qinghai You

amormor@126.com

1 Department of Respiratory and Critical Care Medicine, The First Affiliated Hospital of Anhui Medical University, No. 218 Jixi Road, Hefei 230022, Anhui, China

2 Department of Respiratory Medicine, The Affiliated Hospital of Jining Medical College, No. 89 Guhuai Road, Jining 272029, Shandong, China

3 Department of Respiratory Medicine, The First Affiliated Hospital of Huzhou Teachers College, No. 158 Guangchang Road, Huzhou 313000, Zhejiang, China

4 Department of Respiratory Medicine, The Chest Hospital of Anhui Province, No. 397 Jixi Road, Hefei 230022, Anhui, China

5 Department of Respiratory and Critical Care Medicine, The First Affiliated Hospital of Anhui Medical University, Jixi Road No. 218, Hefei 230022, Anhui, China

\section{Introduction}

Acute respiratory distress syndrome (ARDS) is a severe lung inflammatory disorder commonly characterized by infection or injury inducing the development of diffuse alveolar damage that results in severe hypoxemia. It remains a lethal or disabling medical condition. In intensive care unit (ICU), the incident rate of patients attributed to ARDS is high. The mortality rate of patients with severe ARDS is nearly $50 \%$ as estimated [1]. Even though patients who recover from this disorder are still at high risk for depression, cognitive decline, and persistent skeletal muscle weakness and post-traumatic stress disorder. Risk factors for ARDS include factors that lead to direct or indirect lung injury. For instance, pulmonary contusion, inhalation injury, pneumonia, aspiration of gastric contents and drowning are the common direct lung-injury factors, while pancreatitis, sepsis, major burn injury, drug overdose, transfusion of blood products and cardiopulmonary bypass can indirectly cause lung injury that leads to ARDS. Based 
on the recent clinical findings, more than $85 \%$ of ARDS cases are attributed to sepsis, pneumonia and aspiration of gastric contents [2].

Lung injury-induced inflammatory tissue damage is the core pathogenesis event of ARDS. Initial response to injury is characterized by innate cell-mediated inflammation. The prominent innate cell type activated at this early stage is resident alveolar macrophages that subsequently secrete proinflammatory cytokines or chemokines to promote the activation of alveolar epithelial and endothelial cells and recruitments of monocytes and neutrophils. The subsequent activation of alveolar epithelial and endothelial cells contributes to the damage of barrier function and the accumulation of edema fluid flooding in injured lung tissue. The local accumulation of monocytes and neutrophils produces more inflammatory mediators and results in prolonged tissue damages. Uncontrolled ARDS leads to increased mortality [3].

Though clinical validated biomarkers for ARDS are yet to be defined, common pathological features associated with ARDS include tissue inflammation, alveolar edema and increased lung permeability. Therefore, diffuse alveolar damage is widely recognized as a histological hallmark associated with ARDS [4]. Animal models of ARDS, such as sepsis- or ventilator-induced acute lung injury, have been demonstrated to recapitulate these pathological features in human patients. They are very useful to study the ARDS pathological factors and mechanisms or evaluate the therapeutic factors or agents for managing ARDS [5, 6].

The dysregulated miRNA expression can contribute to the pathogenesis of many diseases including pulmonary disorders, chronic inflammation and cancers [7]. The potential roles of miRNAs in the pathogenesis and progression of ARDS have been reported recently, suggesting a complexity underlying the pathological mechanism of ARDS [8,9]. Elucidating novel miRNA players in ARDS may provide new biomarkers and therapeutic candidates for managing the disorder.

In this study, our aim was to characterize a novel miRNA associated with ARDS and provide scientific insight into therapeutic development to treat ARDS. We used sepsis-induced lung injury as ARDS animal model to profile miRNA expression pattern. The miRNA candidate was further functionally characterized in both in vitro and in vivo models. We also identified and validated a downstream target of the miRNA in ARDS. The established signaling axis revealed a novel molecular mechanism underlying ARDS and provided a new avenue to develop therapeutics for ARDS.

\section{Methods}

\section{Cell culture and treatment}

Primary alveolar macrophages were isolated from lungs by bronchoalveolar lavage and cultured in Dulbecco's modified essential medium (DMEM)/Ham's F-12 medium, supplemented with $10 \%$ fetal bovine serum (FBS), $100 \mathrm{U} /$ $\mathrm{ml}$ streptomycin and $100 \mathrm{U} / \mathrm{ml}$ penicillin. RAW264.7 and A549 were purchased from ATCC and maintained in DMEM or Roswell Park Memorial Institute (RPMI)-1640 medium supplemented with $10 \%$ FBS, $1 \%$ glutamine and antibiotics at $37{ }^{\circ} \mathrm{C}$ and $5 \% \mathrm{CO}_{2}$. Cells were passaged when they reached $80 \%$ confluence. Cells were treated with $1 \mu \mathrm{g} / \mathrm{ml}$ lipopolysaccharides (LPS, Sigma, St. Louis, $\mathrm{MO})$ or DMSO following the time points as indicated in the study. BMS 345541, SB 203580 and SP600125 were purchased from Santa Cruz.

\section{miRNA profiling study}

Total RNA from the sample tissue after the treatment was harvested using Trizol method (Invitrogen, Waltham, MA USA). First-strand cDNA was labelled with Cy3 or Cy5 and used for hybridization reaction on mouse GeneChip miRNA 4.0 array from Thermo Fisher. Fluorescence images were acquired using Affymetrix GCS 3000 scanner. Bioinformatics analysis was performed using the Partek Genomics Suite software. Only fold changes more than two between two groups were considered significant. We included three mice for the control and ARDS group, respectively.

\section{ARDS animal model}

The ARDS mouse model was established by sepsis induction as previously described [10]. Briefly, male BALB/c mice were divided randomly into four groups with 15 subjects in each group: (1) sham control group (receiving saline + scramble control miRNA $20 \mathrm{mg} / \mathrm{kg}$ ); (2) LPS group (receiving LPS $50 \mathrm{mg} / \mathrm{kg}+$ scramble control miRNA $20 \mathrm{mg} /$ $\mathrm{kg}$ ); (3) sham/miRNA group (receiving saline + miRNA802 $20 \mathrm{mg} / \mathrm{kg}$ ); (4) LPS/miRNA group (receiving LPS $50 \mathrm{mg} /$ $\mathrm{kg}+$ miRNA802 $20 \mathrm{mg} / \mathrm{kg}$ ). The miRNAs were delivered using Invivofectamine 3.0 as described previously [11]. $48 \mathrm{~h}$ later, the drugs were delivered by intratracheal instillation. Mice were killed at day 3 upon LPS challenge. The lung tissues were collected for further analysis. The animal study was carried out according to the ethical guidelines approved by Animal Care and Use Committee in the First Affiliated Hospital of Anhui medical University. 


\section{Myeloperoxidase (MPO) assay}

Mice lung tissues were harvested at the end of the study. The neutrophil activation levels in the tissues were quantified by MPO activity assay kit (ab105136) from Abcam following the standard manual. We included eight mice for each group.

\section{Evan's blue staining}

To measure lung permeability, Evan's blue dye $(10 \mathrm{mg} / \mathrm{kg}$, Sigma) was intravenously injected into the mice on the last day of the study. One hour after the injection, the mice were killed and the lungs were harvested and homogenized as previously described [12]. The supernatants containing the dye were collected from the homogenates by centrifugation and quantified in a plate reader at $620 \mathrm{~nm}$ and $740 \mathrm{~nm}$. The corrected value was calculated by Abs 620 (corrected) $=\mathrm{Abs}$ $620-(1.426 \times$ Abs $740+0.030)$. We included eight mice for each group.

\section{TUNEL staining}

Apoptotic cells in the harvested lung tissues were detected by dUTP nick end-labelling (TUNEL) staining kit (Roche Diagnostics) following the manufacturer's manual. The apoptosis rate was calculated as the ratio of TUNEL-positive cells over total cells as stained by DAPI.

\section{Cell transfection}

MiR-802 mimic and scramble control were synthesized by Qiagen. The cells were transfected with the miRNAs using HiPerFect transfection reagent (Qiagen, Valencia, CA, USA) following the manufacturer's manual. Other gene transfections were done with Lipofectamine 2000.

\section{Enzyme-linked immunosorbent assay (ELISA)}

To measure tumor necrosis factor alpha (TNF $\alpha)$ secretion in lung tissue, BALF from the study groups were collected and processed using mouse TNF $\alpha$ ELISA kit from Abcam according to the standard protocol. Peli2 protein expression was determined in homogenized lung tissues using a customized ELISA kit from Cusabio Biotech. We included eight mice for each group.

\section{Luciferase assay}

The 3'UTR of Peli2 containing the wild-type or mutated miR802 target site was cloned into the luciferase reporter plasmid (pMIR-REPORT). Renilla Luciferase vector (pRL-SV40) was used as normalization control. Both miR-802 mimic and luciferase reporter plasmids were co-transfected into BALF macrophage. The luciferase activity was determined $48 \mathrm{~h}$ post-transfection with the Dual Luciferase Assay kit (Promega, Madison, WI, USA) using a plate reader following the manufacture's instruction.

\section{Western blotting}

Cells were harvested in radioimmunoprecipitation assay (RIPA) buffer supplemented with protease inhibitor cocktail (Roche, Penzberg, Upper Bavaria, Germany). $40 \mu \mathrm{g}$ protein lysate was first resolved in $12 \%$ sodium dodecyl sulfate-polyacrylamide gel electrophoresis (SDS-PAGE) and then transferred onto a nitrocellulose membrane. The membrane was blocked with $5 \%$ non-fat milk for $1 \mathrm{~h}$ at room temperature and then incubated with primary antibody (1:1000) for overnight at $4{ }^{\circ} \mathrm{C}$. On the 2 nd day, the membrane was washed with PBST before incubating with a 2nd antibody (1:5000) for $1 \mathrm{~h}$ at room temperature. The target protein was detected using SuperSignal West Pico PLUS chemiluminescent kit. Peli2 and NLRP3 antibodies were from Abcam. Ubiquitin and GAPDH antibodies were purchased from Santa Cruz.

\section{Real-time PCR}

Total RNA including miRNA were harvested using mirVana miRNA isolation kit (Ambion). One microgram of mRNA was converted to cDNA using High-Capacity cDNA Reverse Transcription Kit. And miRNA first-stand cDNA was synthesised using miSCript II RT kit from Qiagen. The gene expression was measured by SYBR Green kit from SigmaAldrich on QuantStudio3 (Applied Biosystems, Waltham, MA, USA) following the standard protocol.

The primer sequences are listed as follows:

$$
\begin{aligned}
& \text { GAPDH (forward): 5' GTGTTCCTACCCCCAATGTG } \\
& \text { 3', } \\
& \text { GAPDH (reverse): 5' CATCGAAGGTGGAAGAGTGG } \\
& \text { 3', } \\
& \text { TNF } \alpha \text { (forward): 5' CGTCAGCCGATTTGCTATCT 3', } \\
& \text { TNF } \alpha \text { (reverse): 5' CTTGGGCAGATTGACCTCAG 3', } \\
& \text { IL-1 } \beta \text { (forward): 5' GGGCCTCAAAGGAAAGAATC 3', } \\
& \text { IL-1 } \beta \text { (reverse): 5' TACCAGTTGGGGAACTCTGC 3', } \\
& \text { IL-6 (forward): 5' GGGACTGATGCTGGTGACAA 3', } \\
& \text { IL-6 (reverse): 5' TCCACGATTTCCCAGAGAACA 3', } \\
& \text { U6 (forward): 5' CTCGCTTCGGCAGCACA 3', } \\
& \text { U6 (reverse): 5' AACGCTTCACGAATTTGCGT 3', } \\
& \text { miRNA802 (forward): 5' TCCAGTGCCAGAAATGAA } \\
& \text { CC 3', } \\
& \text { miRNA802 (reverse): 5' CTGAACCACAGTTACAGA } \\
& \text { GC 3'. }
\end{aligned}
$$




\section{Statistical analysis}

The statistical analysis was done using SPSS software. The data were presented as mean \pm standard deviation (SD). The unpaired Student's $t$ test was employed to compare the differences between the groups. One-way ANOVA analysis followed by a Tukey's post hoc test was applied to the comparison of more than two groups. Only $p$ value less than 0.05 was considered significant.

\section{Results}

\section{miR-802 is downregulated in ARDS model}

To elucidate the miRNA candidates implicated in the pathogenesis of ARDS, we profiled the expression changes of miRNAs in a LPS-induced ARDS mouse model. LPS was administrated intratracheally to induce acute lung injury. $24 \mathrm{~h}$ later, the lung tissues were harvested and processed for microarray analysis of miRNA gene expression. A list of miRNAs showing most significant changes is shown in Fig. 1a. Among those, miR-802 was one of the most significantly downregulated targets in ARDS lung tissue. The role of this candidate in ARDS or other lung injury has not been reported, which made it a novel miRNA in ARDS field. To explore its function in ARDS, we subsequently isolated alveolar macrophages from lungs by bronchoalveolar lavage. The primary macrophages were cultured and challenged with LPS. We found LPS stimulation reduced the expression of miR-802 to almost fourfold (Fig. 1b). This was consistent with the in vivo result. We also confirmed the LPS-mediated suppression of miR-802 in RAW264.7, an immortalized monocyte/macrophage line (Fig. 1c). Interestingly, when we challenged A549, an alveolar basal epithelial cell line, no miR-802 reduction was detected (Fig. 1d). Therefore, miR802 in lung tissue was suppressed by LPS and the reduction was largely attributed to the response in macrophages.

\section{miR-802 suppresses inflammatory cytokine production}

Next, we proceeded to elucidate the role of miR-802 in LPS-induced lung injury. Macrophages are a key cell type in lung in response to LPS challenge and proinflammatory cytokine production is a critical step that mediates LPSinduced tissue damage. Since miR-802 was found reduced by LPS in alveolar macrophages, we restored the miRNA expression in the cells and examines its impact on proinflammatory response induced by LPS. As shown (Fig. 2a), macrophages challenged with LPS produced high level of $\mathrm{TNF} \alpha$, while overexpressing miR-802 before LPS stimulation greatly suppressed the TNF $\alpha$ expression. We observed similar suppressing effects of miR-802 in LPS-mediated interleukin-1 beta (IL-1 $\beta$ ) and IL-6 induction (Fig. 2b, c). Therefore, we concluded miR-802 expression may antagonize proinflammatory cytokine production during LPSmediated lung inflammatory response and tissue damage. Independently prolonged LPS challenge could induce cell apoptosis. As shown (Fig. 2d), LPS increased cell death rate in macrophages as measured by TUNEL assay. However, miR-802 expression did not change the LPS effect on cell apoptosis. Therefore, the regulatory function of miR-802 may be specific to inflammatory pathway.

\section{miR-802 alleviates lung damages in ARDS model}

To evaluate the effect of miR-802 on LPS-induced lung acute injury in vivo, the mice were administrated with miR802 or scramble control intragastrically before being subjected to sepsis challenge. First, we analyzed the morphological change of lung tissues with H\&E staining. As shown (Fig. 3a), sepsis group showed increasing neutrophil infiltration, alveolar hemorrhage, lung edema, and compromised epithelial/endothelial cell structure compared with the sham group. Interestingly pre-treatment of miR-802 significantly reduced tissue damage and led to a significant improvement on lung morphology. Second, we determined the neutrophils activation by measuring myeloperoxidase (MPO) activity in lung tissues. As shown (Fig. 3b), MPO activity increased greatly in LPS-challenged lung tissues and the delivery of miR-802 reduced the increased MPO activity in damaged lung. LPS challenge also increased the permeability of the lung tissues as measured by Evan's blue staining (Fig. 3c). In miR-802-treated group, the LPS-mediated protein leakage in lung tissues was significantly improved (Fig. 3c). Last but not least, since the suppression effect of miR-802 in inflammatory cytokine production was found in vitro, we proceeded the measure level in the lung tissues. As expected, LPS administration caused a significant induction of TNF $\alpha$ in the damaged tissues and overexpressing miR-802 markedly antagonized the accumulation of the proinflammatory factor (Fig. 3d). Taken together, the in vivo study suggested a protective role of miR-802 in LPS-induced ARDS model.

\section{Peli2 is a direct target of $\mathbf{m i R - 8 0 2 ~ i n ~ c e l l s ~}$}

Next, we sought to identify the downstream target of miR802 in lung macrophages. We analyzed the putative targets of miR-802 in miRBase [13]. Among them Peli2 carried a miR-802 target site in its 3'UTR, which was conserved in both mouse and human (Fig. 4a). Peli2 is an E3 ligase that mediates the inflammatory response in response to LPS [14]. We hypothesized that miR-802 may target Peli2 to suppress LPS challenge. Therefore, we constructed luciferase reporter gene (denoted as WT), in which Peli2 
A

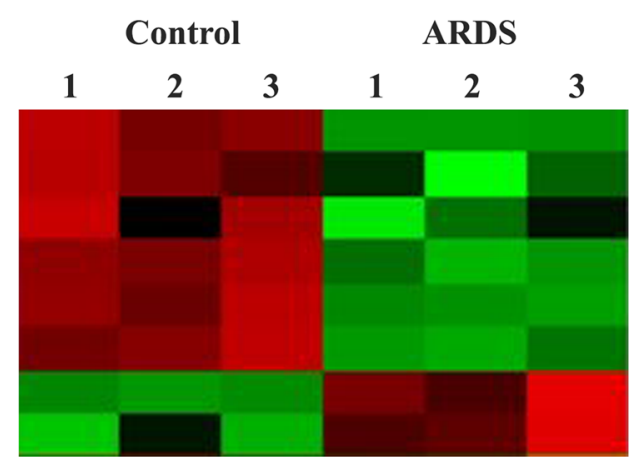

miR-802

miR-520

miR-5121

miR-155

miR-300

miR-96

miR-199a

miR-200b

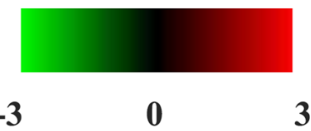

C

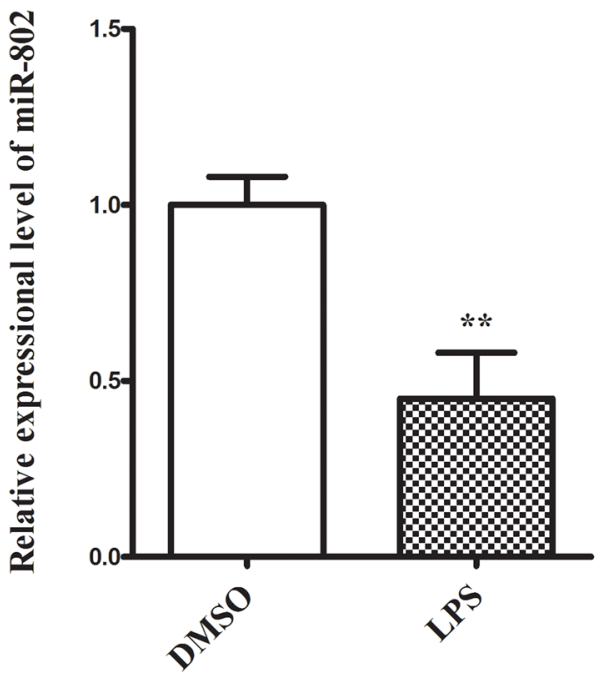

Fig. 1 miR-802 is in downregulated in LPS induced ARDS model. a Lung tissues from ARDS or control group were harvested. The miRNA expression profiles were analyzed by GeneChip ${ }^{\mathrm{TM}}$ miRNA 4.0 Array. A list of the miRNAs including miR-802 showing most significant changes in ARDS group was shown. b Primary lung mac-

3'UTR containing the miRNA target site was cloned to the downstream of the coding sequence. As comparison, we constructed the MUT vector in which the miRNA target site in Peli2 3'UTR was mutated (Fig. 4a). To access the binding, we co-transfected the WT vector with either miR-802 or scramble control. As shown (Fig. 4b), miR802 but not scramble miRNA markedly reduced the luciferase activity. In contrast, co-transfection of miR-802 with the MUT vector did not impact on the luciferase activity (Fig. 4b). To confirm the direct regulation of miR-802 on
B

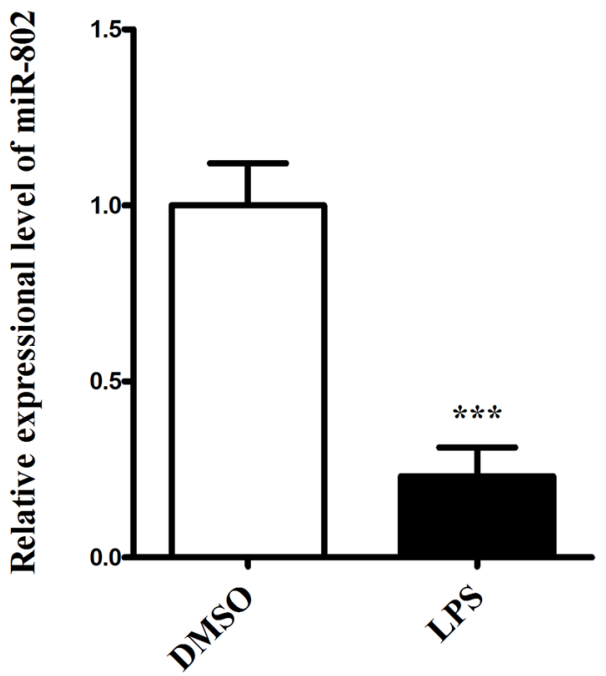

D



rophages, c RAW264.7, and d A547 cells were challenged with LPS $(1 \mu \mathrm{g} / \mathrm{ml})$ for $24 \mathrm{~h}$. The expression change of miR-802 was analyzed by real-time PCR. Data were presented as mean \pm SD. ${ }^{* *} p<0.01$, $* * * p<0.001$, compared with DMSO control

Peli2, we expressed either the miRNA or the scramble control in alveolar macrophages and then measured Peli2 protein level. As shown (Fig. 4c), overexpression of miR802 markedly decreased the protein expression of Peli2. Functionally Peli2 controls the activation NLRP3 inflammasome by promoting its ubiquitination in response to LPS [15]. Thus, we proceeded to measure the effect of miR-802 in NLRP3 activation by LPS. As shown (Fig. 4d), LPS stimulation promoted the accumulation of K63-linked ubiquitinated NLRP3, which was an indicator of NLRP3 
Fig. 2 miR-802 suppresses LPS-induced inflammatory cytokine production. Primary lung macrophages were transfected with miR-802 mimic or scramble control $48 \mathrm{~h}$ before LPS challenge. $24 \mathrm{~h}$ after LPS stimulation, cells were harvested for mRNA extraction. The gene expression levels of a TNF $\alpha$, b IL- $1 \beta$ and $\mathbf{c ~ I L - 6 ~ w e r e ~}$ quantified by real-time PCR. d $48 \mathrm{~h}$ after LPS challenge, the apoptotic cells from each condition were measured by TUNEL assay. Data were presented as mean \pm SD. Independent experiments were repeated in triplicate. ${ }^{*} p<0.01, * * * p<0.001$, compared with DMSO control;

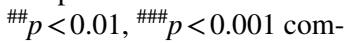
pared with scramble control in the same treatment condition
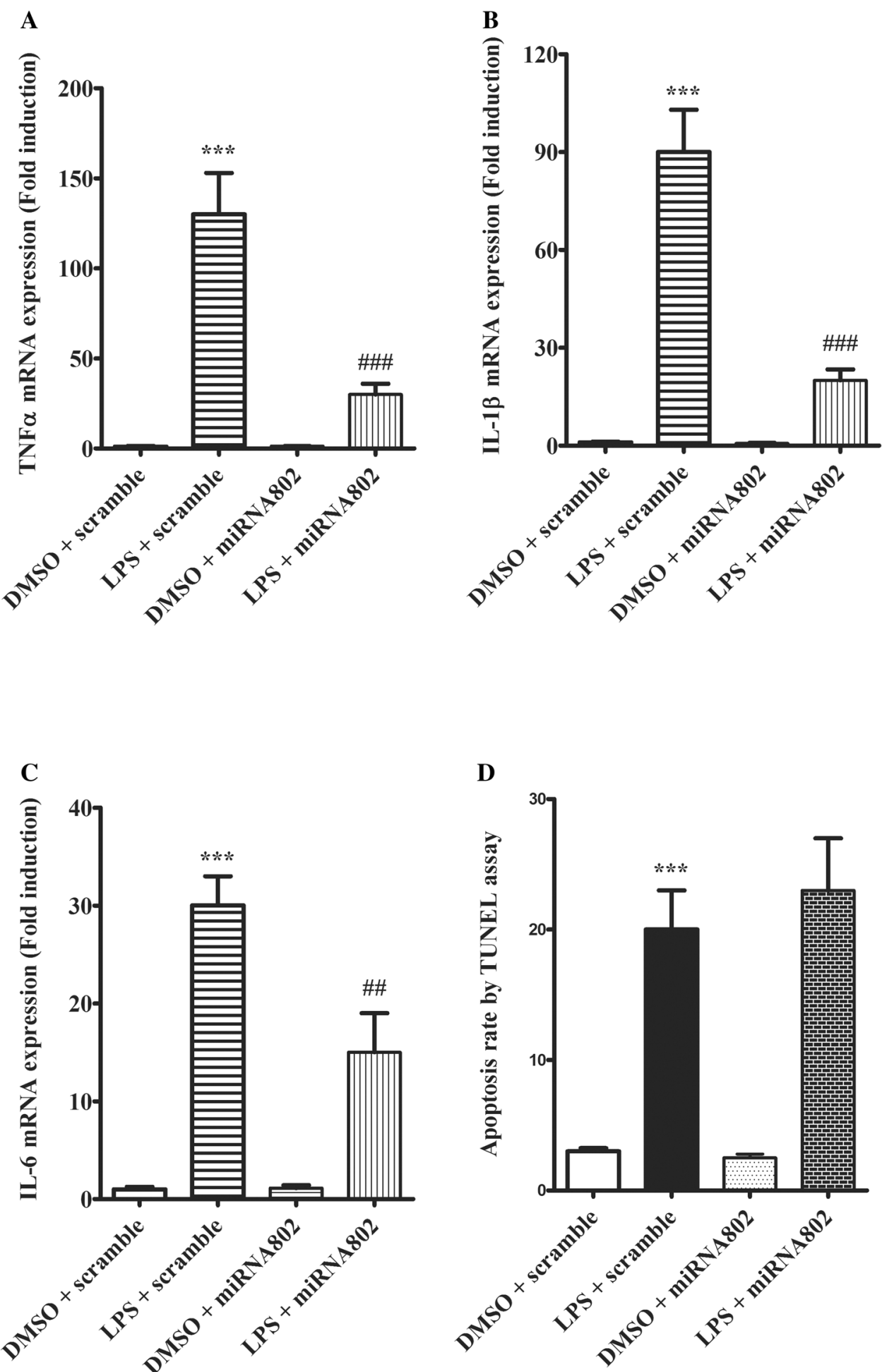

activation. Interestingly, overexpressing miR-802 abrogated the ubiquitination on NLRP3, which may be attributed to the downregulation of Peli2 by the miRNA. Taken together, we identified Peli2 was a direct downstream target of miR-802 in lung macrophages.

\section{Peli2 antagonizes miR-802-mediated suppression on LPS-induced proinflammatory response}

Next, we sought to confirm the functional relevance of Peli2 in miR-802-mediated inflammation suppression in 
Fig. 3 miR-802 reduces lung damages in ARDS model. a The effect of miR-802 mimic on LPS-induced histological changes in lung tissues $(n=8$ for each group). $\mathbf{b}$ The effect of miR-802 mimic on neutrophil activation in damaged lung tissues. The activation was measured by MPO activity. c The effect of miR-802 mimic on LPS-induced tissue permeability damage as measured by Evan's blue staining. $\mathbf{d}$ The effect of miR-802 mimic on LPS-induced TNF $\alpha$ production. The level of TNF $\alpha$ was quantified by ELISA. Independent experiments were repeated in triplicate. $*<0.05, * * p<0.01$, $* * * p<0.001$, compared with sham treatment group; ${ }^{\#}<0.05$,

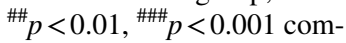
pared with scramble control receiving same treatment
A

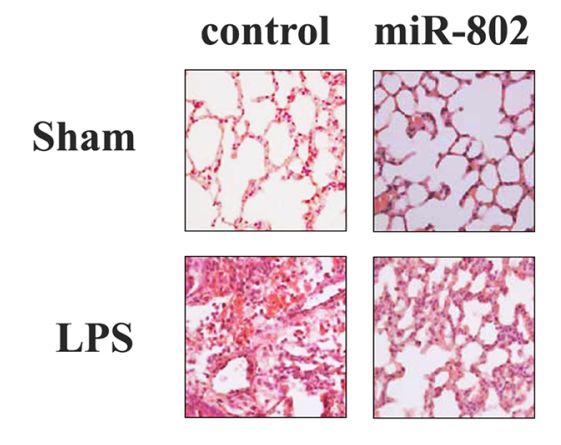

C

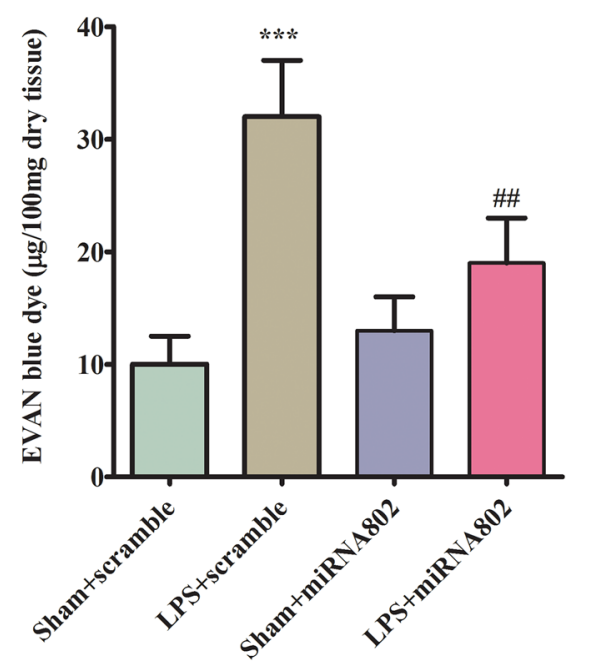

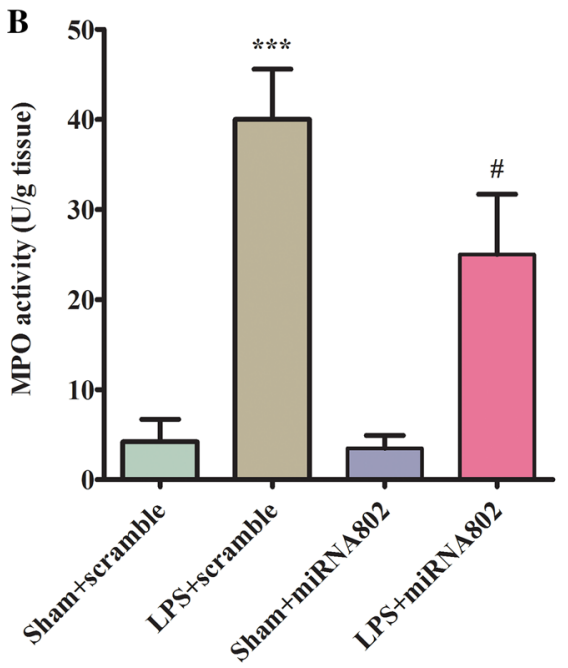

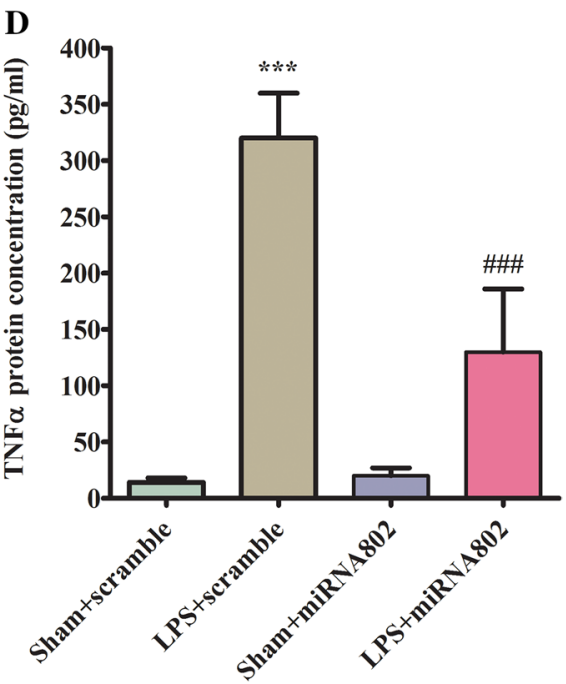

ARDS model. In ARDS mouse model, miR-802 was found downregulated. As the hypothetical target of the miRNA, Peli2 level would increase in response to LPS challenge in lung tissues. We then compared Peli2 protein expression in lung samples between LPS or sham groups. As shown (Fig. 5a), as expected, Peli2 protein concentration was significantly higher in LPS group. In the rescue study, we examined the effect of Peli2 in miR-802-mediated inflammation inhibition in primary macrophages with LPS challenge. As shown (Fig. 5b), co-transfection of Peli2 with miR-802 abolished the antagonizing effect of the miRNA on LPS-induced TNF $\alpha$ expression. Similarly, overexpression of Peli2 was also found to abrogate the inhibition of miR- 802 on IL- $1 \beta$ and IL- 6 production primed by LPS (Fig. 5c, d). Overall, these results indicated miR-802 targeted Peli2 to suppress LPS-induced lung inflammation reaction.

\section{LPS-mediated miR802 suppression is NFKB pathway dependent}

Last but not least, we explored the mechanistic regulation of LPS on miR802 suppression. LPS binds to Toll-like receptor 4 (TLR4) that leads to TAK1 activation. TAK1 is a pivotal regulator that subsequently activates nuclear factor kappa-light-chain enhancer of activated B cells (NFkB), p38 and c-Jun N-terminal kinase (JNK) sub-pathways [16]. We treated primary macrophages with specific inhibitors targeting these downstream factors, including BMS345541, a


SB203580 and SP600125 that directly target p38 and JNK, respectively. After $24 \mathrm{~h}$, we challenged the cells with LPS and measured the effect on miR802 suppression. As shown in Fig. 6a, only BMS345541 but not the other two inhibitors could disrupt the suppression effect on the miRNA. 


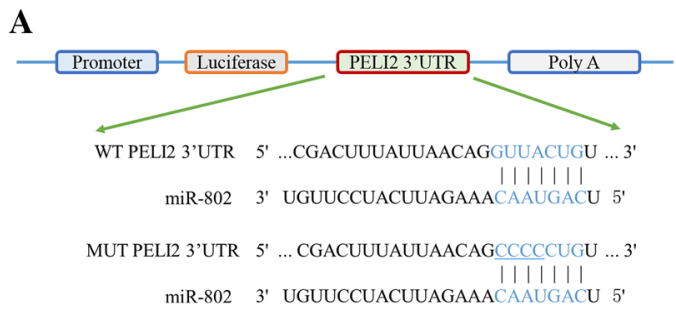

C

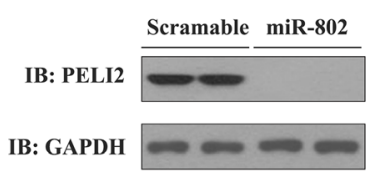

Fig. 4 Peli2 is a direct target of miR-802 in cells. a Schematic diagram of a conserved putative miR-802 targeting site in 3'UTR of Peli2 (WT). The site mutations were introduced to miR-802 targeting site of 3'UTR of Peli2 (MUT). b Luciferase reporter assay study of the interaction between miR-802 and 3'UTR of Peli2. Lung macrophages were first transfected with luciferase reporter genes conjugated with either wild-type (WT) or mutant (MUT) 3'UTR of Peli2. After $24 \mathrm{~h}$, the cells were transfected with either miR-802 mimic or scramble control. Transfected cells were cultured for another $48 \mathrm{~h}$ before the measurement of luciferase activities in a plate reader. c Western blotting analysis of Peli2 protein expression in cells trans-

To confirm this, we also repeated the same treatment on RAW264.7. Consistently only NFKB could specifically abolish LPS-mediated miR802 reduction (Fig. 6b). Taken together LPS-induced miR802 suppression was NFkB pathway dependent (Fig. 6c).

\section{Discussion}

With miRNA profiling, we identified a list of candidates that may play parts in ARDS model. At the top of the list, miR802, miR-520, miR-5121, miR-155, miR-300, and miR-96 are found significantly downregulated in the disease model.
B
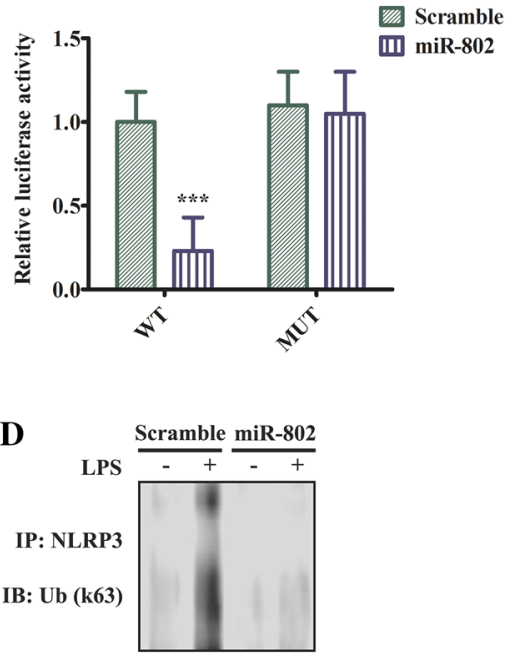

IP: NLRP3 IB: NLRP3

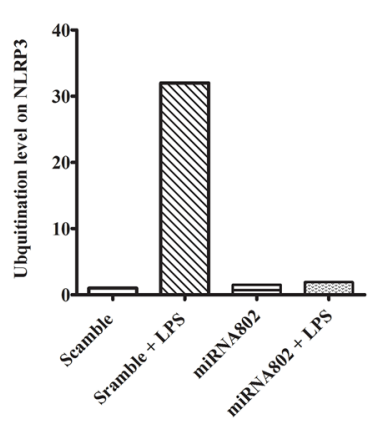

fected with miR-802 or scramble control. GAPDH was used as loading control. The normalized Peli2 expression was quantified by densitometry. d Western blotting analysis of NLRP3 ubiquitination in cells transfected with miR-802 or scramble control. NLRP3 protein was pulled down with a monoclonal antibody. The immunoprecipitated complex was resolve in SDS-PAGE and immunoblotted with ubiquitin antibody. The ubiquitination level on Peli2 was quantified by densitometry. Data were expressed as mean $\pm \mathrm{SD}$. Independent experiments were repeated in triplicate. $* * * p<0.001$ significantly different from control group

The upregulated candidates are miR-199a and miR-200b. In this study, we have elucidated the role of miR-802 in ARDS. Specifically, the miRNA downregulation has been confirmed in LPS-challenged lung macrophages but not epithelial cells. Restoring the miRNA expressing level in LPS-challenged macrophages can reduce the overproduction of proinflammatory cytokines such as TNF $\alpha$, IL- $1 \beta$ and IL- 6 . However, the overexpression of miR-802 cannot inhibit the cell death challenged by LPS, suggesting the regulation of miR-802 in macrophages may be specific to proinflammatory pathway. The benefits of overexpressing miR-802 in ARDS can be extended to the in vivo model. We have observed the alleviation in neutrophil activation, tissue permeability and 
Fig. 5 Peli2 expression antagonizes miR-802-mediated suppression of LPS-induced proinflammatory response.

a The protein expressions of Peli2 in lung tissues between LPS-induced ARDS model and sham group were compared by ELISA analysis $(n=15$, for each group). The primary lung macrophages were co-transfected with miRNA or Peli2 before LPS challenge. $24 \mathrm{~h}$ later, the gene expression levels of $\mathbf{b}$ TNF $\alpha$, c IL- $1 \beta$ and $\mathbf{d}$ IL- 6 with or without LPS stimulation were quantified by real-time PCR. Independent experiments were repeated in triplicate. $* * p<0.01, * * * p<0.001$, compared with DMSO control; ${ }^{\# \#} p<0.01$, \#\#\# $p<0.001$ compared with no Peli2 control in the same treatment condition
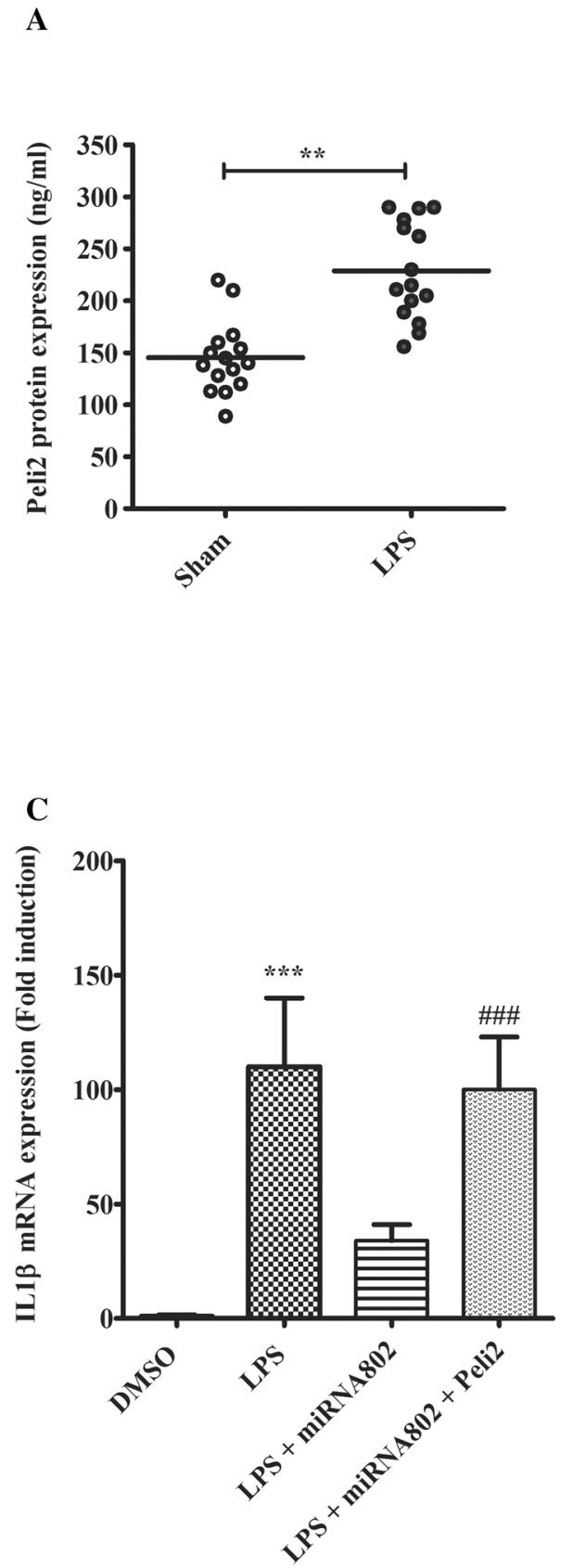
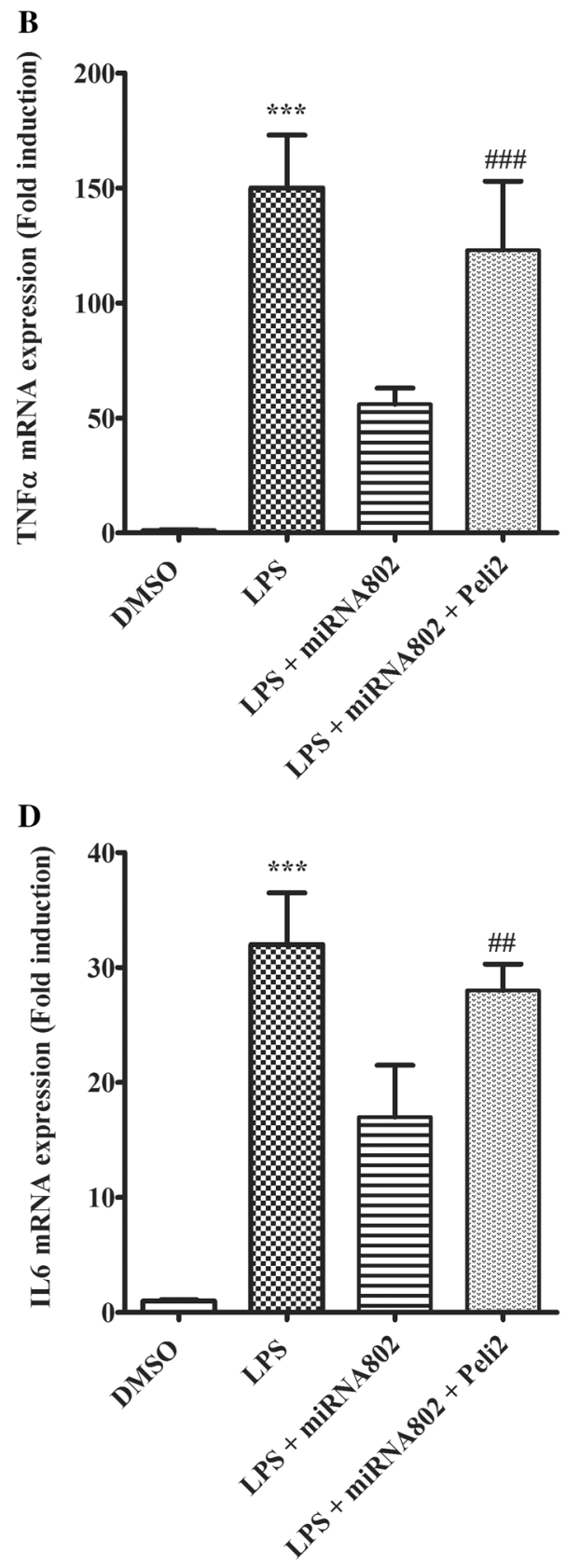

proinflammatory cytokine secretion in addition to the clinical improvements by histological analysis. In the same study, we have identified Peli2, a positive regulator in LPS/TLR4 pathway, as downstream target of miR-802 in macrophages. Overexpressing miR-802 can repress Peli2 expression and NLRP3 ubiquitination. Interestingly, Peli2 expression has been found upregulated in ARDS model that inversely correlates with the miR-802 expression. More importantly, coexpression of Peli2 in miR-802-transfected macrophages can abolish the antagonizing effect of the miRNA in proinflammatory pathway. Last but not least, inhibiting NFKB activation but not $\mathrm{p} 38$ or JNK phosphorylation specifically abolishes LPS-mediated repression of miR802 expression. Therefore, our study has shown strong evidence to support the involvement of miR-802/Peli2 signalling axis in ARDS (Fig. 6c).

Acute respiratory distress syndrome as a severe form of lung injury is commonly found in critically ill patients. Lack of effective therapeutic targets for ARDS leads to the high mortality rate of ARDS patients. MiRNAs are emerging as promising drug targets for many diseases. They can regulate the singling proteins post-transcriptionally to control the disease progression. In ARDS, modified expression of miRNAs has been studied to develop the diagnosis and treatment for 

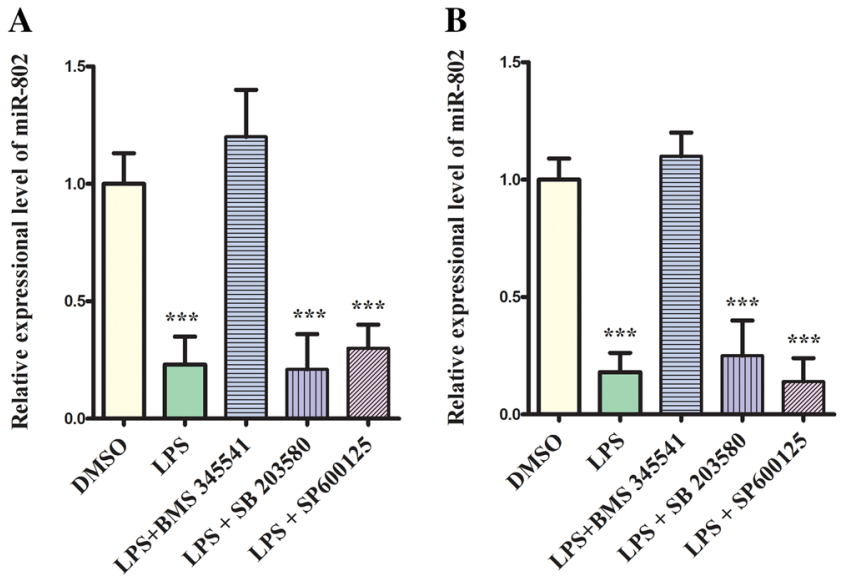

C

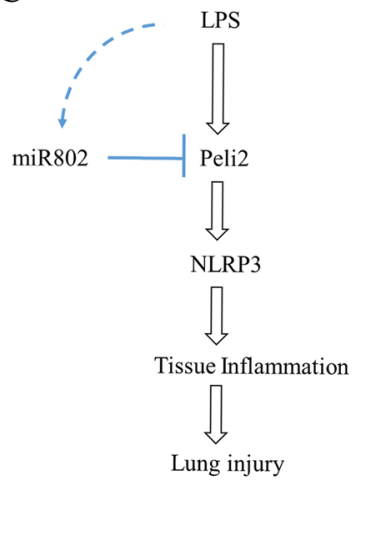

Fig. 6 LPS-mediated miR802 suppression was through NFkB pathway. a Primary macrophages or b RAW264.7 cells were incubated with $1 \mu \mathrm{M}$ each of the following inhibitors for overnight. On the next day, the cells were challenged with $1 \mu \mathrm{g} / \mathrm{ml}$ LPS for overnight and harvested to measure miR802 expression. The representative

the diseases $[17,18]$. Microarray-based miRNA profiling has been employed to study the modified miRNA expression in rat ARDS model [8]. Many miRNAs involved in the pathogenesis of ARDS regulate either tissue repair or inflammatory response in lung tissue. In LPS-induced ARDS model, miR-233 has been found downregulated with a similar trend as miR-802 in our study. Overexpressing miR-233 can attenuate LPS-mediated inflammation in the disease model. Interestingly, miR-233 has been also found to inhibit NLRP3 inflammasome by targeting rho-related GTPbinding protein RhoB (RHOB) [19]. In another study, miR155 has been reported being elevated in bronchoalveolar lavage fluid (BALF) samples of ARDS patients. In this case, the elevation of the miRNA is likely an adaptive mechanism to the stress. In the animal experiment, the overexpression of miR-155 alleviates septic lung injury by targeting transforming growth factor- $\beta$-activated binding protein 2 (TAB2), a regulatory molecule under TLR4 signalling [20]. In another similar study, miR-146a has been characterized as a suppressor in LPS-induced acute lung injury [21]. The upregulation of miR-146a inhibits the inflammatory responses by suppressing the expression of TRAF-6 and IRAK-1. Interestingly, IRAK1 is acting upstream of Peli2 under TLR4 activation. The activation of Peli2 by IRAK1 enhances its E3 ligase activity. The activated Peli2 mediates the ubiquitination and activation of NLRP3 inflammasome [15, 22]. There are increasing evidences from ARDS animal models supporting this signalling axis can be regulated by multiple miRNAs including miR-802 from our study. These studies indicate TLR4 signalling be critical for the initiation and development of acute lung injury and the signalling components in the pathway carrying the therapeutic potential for managing ARDS. results from at least three biological repeats were shown. Data were expressed as mean $\pm \mathrm{SD}$. Independent experiments were repeated in triplicate. ${ }^{*} p<0.01, * * * p<0.001$, compared with DMSO control. c Schematic diagram showing the biological role of miR802 in LPSinduced ARDS model as supported in our study

miR-802 has been studied intensively in cancer biology. Generally, it has been proposed as a tumor suppressor. For instance, in cervical cancer, miR-802 was downregulated and the restoration of the miRNA inhibits serine-/argininerich splicing factor9 (SRSF9) to induce cancer cell apoptosis [23]. The enforced expression of miR-802 can antagonize gastric cancer oncogenesis by inhibiting RAB23 expression [24]. Similarly, miR-802 can also act as a tumor suppressor against tongue squamous cell carcinoma growth and metastasis [25]. In tongue squamous cells, miR-802 directly regulates the expression of MAP2K4. In addition, miR-802 has been demonstrated in human prostate cancer to regulate epithelial-mesenchymal transition process by targeting flotillin-2 [26]. Therefore, the role of miR-802 is cell type specific and depends on the molecular targets acting downstream. Our study reveals that the miRNA has a novel role in controlling inflammatory response. In lung tissue particularly lung macrophages, miR- 802 targets Peli2 in TLR4 pathway. The enforced expression of miR- 802 seems unlikely to induce any unwanted risks such as oncogenesis. Therefore, targeting miR-802/Peli2 axis holds a promise to develop therapeutics for ARDS.

Currently, the first-line treatment for severe ARDS is extracorporeal membrane oxygenation (ECMO) or extracorporeal $\mathrm{CO}_{2}$ elimination [27]. Early recognition and targeting intervention are crucial to improve the clinical outcomes of ARDS treatment in the future. Newly identified miR-802/ Peli2 may shed light on precise intervention therapy.

To test the validity of miR-802/Peli2 in treating ARDS, it will be important to confirm the miRNA expression change in human patients for the next step. Furthermore, using siRNA or small molecule inhibitor to target Peli2 in animal ARDS model will also be planned to evaluate whether the 
axis is critical for the pathogenesis of ARDS. However, our study only focuses on sterile inflammatory ARDS model induced by LPS. It will be important to evaluate the role of miR-802 in other mouse model of ARDS, such as an infectious model driven by Streptococcus pneumoniae or ventilator-induced acute lung injury $[5,6]$. In addition, to be more clinically relevant, it is also critical in the future to validate the expression pattern of miR-802/Peli2 in human ARDS patients before developing the intervention targeting strategy on this promising pathway.

\section{Conclusion}

In conclusion, miR-802 restoration or Peli2 inhibition may provide a new avenue to treat ARDS.

Funding The study was supported by the National Natural Science Foundation of China (81100053).

\section{Compliance with ethical standards}

Conflict of interest The authors declare no conflicts of interest.

Ethical approval The animal study was carried out according to the ethical guidelines approved by Animal Care and Use Committee in the First Affiliated Hospital of Anhui medical University.

\section{References}

1. Force ADT, Ranieri VM, Rubenfeld GD, Thompson BT, Ferguson $\mathrm{ND}$, Caldwell E, et al. Acute respiratory distress syndrome: the Berlin definition. JAMA. 2012;307(23):2526-33.

2. Ware LB, Matthay MA. The acute respiratory distress syndrome. N Engl J Med. 2000;342(18):1334-499.

3. Butt Y, Kurdowska A, Allen TC. Acute lung injury: a clinical and molecular review. Arch Pathol Lab Med. 2016;140(4):345-50.

4. Matthay MA, Ware LB, Zimmerman GA. The acute respiratory distress syndrome. J Clin Invest. 2012;122(8):2731-40.

5. Aeffner F, Bolon B, Davis IC. Mouse models of acute respiratory distress syndrome: a review of analytical approaches, pathologic features, and common measurements. Toxicol Pathol. 2015;43(8):1074-92.

6. Bastarache JA, Blackwell TS. Development of animal models for the acute respiratory distress syndrome. Dis Model Mech. 2009;2(5-6):218-23.

7. Bushati N, Cohen SM. microRNA functions. Annu Rev Cell Dev Biol. 2007;23:175-205.

8. Guan Y, Jin X, Liu X, Huang Y, Wang M, Li X. Identification of microRNAs in acute respiratory distress syndrome based on microRNA expression profile in rats. Mol Med Rep. 2017;16(3):3357-62.

9. Huang C, Xiao X, Chintagari NR, Breshears M, Wang Y, Liu L. MicroRNA and mRNA expression profiling in rat acute respiratory distress syndrome. BMC Med Genom. 2014;7:46.

10. Li W, Qiu X, Jiang H, Han Y, Wei D, Liu J. Downregulation of miR-181a protects mice from LPS-induced acute lung injury by targeting Bcl-2. Biomed Pharmacother. 2016;84:1375-82.
11. Brown RAM, Richardson KL, Kalinowski FC, Epis MR, Horsham JL, Kabir TD, et al. Evaluation of MicroRNA delivery in vivo. Methods Mol Biol. 2018;16(99):155-78.

12. Liu Y, Guan H, Zhang JL, Zheng Z, Wang HT, Tao K, et al. Acute downregulation of miR-199a attenuates sepsis-induced acute lung injury by targeting SIRT1. Am J Physiol Cell Physiol. 2018;314(4):C449-C45555.

13. Griffiths-Jones S, Grocock RJ, van Dongen S, Bateman A, Enright AJ. miRBase: microRNA sequences, targets and gene nomenclature. Nucleic Acids Res. 2006;34(Database issue):D140-D144144.

14. Butler MP, Hanly JA, Moynagh PN. Kinase-active interleukin-1 receptor-associated kinases promote polyubiquitination and degradation of the Pellino family: direct evidence for PELLINO proteins being ubiquitin-protein isopeptide ligases. J Biol Chem. 2007;282(41):29729-37.

15. Humphries F, Bergin R, Jackson R, Delagic N, Wang B, Yang S, et al. The E3 ubiquitin ligase Pellino2 mediates priming of the NLRP3 inflammasome. Nat Commun. 2018;9(1):1560.

16. Dai L, Aye Thu C, Liu XY, Xi J, Cheung PC. TAK1, more than just innate immunity. IUBMB Life. 2012;64(10):825-34.

17. Ferruelo A, Penuelas O, Lorente JA. MicroRNAs as biomarkers of acute lung injury. Ann Transl Med. 2018;6(2):34.

18. Rajasekaran S, Pattarayan D, Rajaguru P, Sudhakar Gandhi PS, Thimmulappa RK. MicroRNA regulation of acute lung injury and acute respiratory distress syndrome. J Cell Physiol. 2016;231(10):2097-106.

19. Yan Y, Lu K, Ye T, Zhang Z. MicroRNA223 attenuates LPSinduced inflammation in an acute lung injury model via the NLRP3 inflammasome and TLR4/NFkappaB signaling pathway via RHOB. Int J Mol Med. 2019;43(3):1467-77.

20. Liu F, Nie C, Zhao N, Wang Y, Liu Y, Li Y, et al. MiR-155 alleviates septic lung injury by inducing autophagy via inhibition of transforming growth factor-beta-activated binding protein 2 . Shock. 2017;48(1):61-8.

21. Zeng Z, Gong H, Li Y, Jie K, Ding C, Shao Q, et al. Upregulation of miR-146a contributes to the suppression of inflammatory responses in LPS-induced acute lung injury. Exp Lung Res. 2013;39(7):275-82.

22. Lin CC, Huoh YS, Schmitz KR, Jensen LE, Ferguson KM. Pellino proteins contain a cryptic FHA domain that mediates interaction with phosphorylated IRAK1. Structure. 2008;16(12):1806-16.

23. Zhang Q, Lv R, Guo W, Li X. microRNA-802 inhibits cell proliferation and induces apoptosis in human cervical cancer by targeting serine/arginine-rich splicing factor 9. J Cell Biochem. 2019;120(6):10370-9.

24. Zhang XY, Mu JH, Liu LY, Zhang HZ. Upregulation of miR802 suppresses gastric cancer oncogenicity via targeting RAB23 expression. Eur Rev Med Pharmacol Sci. 2017;21(18):4071-8.

25. Wu X, Gong Z, Sun L, Ma L, Wang Q. MicroRNA-802 plays a tumour suppressive role in tongue squamous cell carcinoma through directly targeting MAP2K4. Cell Prolif. 2017;50(3):e12336.

26. Wang D, Lu G, Shao Y, Xu D. microRNA-802 inhibits epithelialmesenchymal transition through targeting flotillin-2 in human prostate cancer. Biosci Rep. 2017;37(2):BSR20160521.

27. Pan C, Liu L, Xie JF, Qiu HB. Acute respiratory distress syndrome: challenge for diagnosis and therapy. Chin Med J (Engl). 2018;131(10):1220-4.

Publisher's Note Springer Nature remains neutral with regard to jurisdictional claims in published maps and institutional affiliations. 